\title{
Characteristics Associated with Negative Interferon-y Release Assay Results in Culture-Confirmed Tuberculosis Patients, Texas, USA, 2013-2015
}

\author{
Duc T. Nguyen, Larry D. Teeter, Julie Graves, Edward A. Graviss
}

Interferon- $\gamma$ release assays (IGRAs) are the preferred diagnostic test for tuberculosis (TB) infection in at-risk populations in developed countries. However, IGRAs have high false-negative rates in patients with TB disease. Populationbased studies assessing the factors associated with negative IGRA results in TB patients have not been performed. Using statewide TB surveillance data of culture-confirmed TB patients in Texas, USA, during 2013-2015, we describe the patient characteristics and treatment outcomes associated with false-negative IGRA results. Among 2,854 TB patients, 1,527 $(53.5 \%)$ had an IGRA result; $97.4 \%(1,487 / 1,527)$ of those had a positive $(87.7 \%)$ or negative $(12.3 \%)$ result. Older age, HIV co-infection, non-Hispanic white race/ethnicity, and being tested with T-SPOT.TB were associated with negative IGRA results. TB patients with negative IGRA results had a higher mortality, potentially due to delayed treatment. Healthcare providers should consider these risk factors when making decisions for patients with suspected TB and negative IGRA results and potentially provide treatment.

Tnterferon- $\gamma$ release assays (IGRAs) are blood tests that 1 measure immune reactivity to Mycobacterium tuberculosis-specific antigens and aid in the diagnosis of latent tuberculosis (TB) infection (LTBI). During the period of this study, 2 IGRAs, which have been approved by the US Food and Drug Administration, were commercially available for use in the United States: QuantiFERON-TB Gold In-Tube (QFT; QIAGEN, Germantown, MD, USA); and T-SPOT.TB (Oxford Immunotec, Inc., Marlborough, MA, USA) (1). In Texas, a state with one of the highest TB burdens in the United States, IGRAs have been used

Author affiliations: Houston Methodist Research Institute, Houston, Texas, USA (D.T. Nguyen, E.A. Graviss); Forensic Research and Analysis, Portland, Oregon, USA (L.D. Teeter); University of Medicine and Health Sciences, New York, New York, USA (J. Graves); University of Texas School of Public Health, Houston (J. Graves)

DOI: https://doi.org/10.3201/eid2403.171633 in targeted testing among persons at high risk for LTBI or at high risk of developing TB disease once infected with TB (2). Although IGRAs are not recommended to be used as rule-out tests for TB disease because of their inability to differentiate LTBI from TB disease and inconsistent sensitivity and specificity in different populations with TB disease (3-7), IGRAs are still preferred tools used by healthcare providers to identify LTBI in persons being evaluated for TB disease before biological confirmation. In the absence of other positive rapid diagnostic test results for TB disease, a negative IGRA result might inappropriately lower the clinical suspicion for TB and result in delayed treatment initiation. Previous studies have suggested that older age, underweight, HIV co-infection, extrapulmonary TB, and increased number of human leukocyte antigen DRB1*0701 alleles were associated with negative IGRA results $(8-11)$. However, these studies had small sample sizes or were performed at a single center. In addition, little information is available on deaths associated with negative IGRA results in a population with confirmed TB. The main objectives of our population-based analysis were to identify the demographics, clinical characteristics, and patient outcomes associated with negative IGRA results in patients with culture-confirmed TB.

\section{Methods}

We acquired deidentified TB surveillance data of patients with TB disease reported in the Texas National Electronic Disease Surveillance System (NEDSS) database during January 1, 2013-December 31, 2015. NEDSS is a TB surveillance data system used by all state jurisdictions within the United States to facilitate the electronic transfer of public health surveillance data from the healthcare system to public health departments (12). The downloaded data included sociodemographic, clinical, laboratory, and radiographic characteristics. We downloaded genotype information from the Centers for Disease Control and Prevention TB 
Genotyping Information Management System (13). We included for analysis only the TB patients who were confirmed TB positive by $M$. tuberculosis culture and had either a positive or negative IGRA result available. We excluded TB patients with negative culture results and those for whom specimens were not taken for TB culture (clinical TB patients), as well as those with unavailable or unknown IGRA types or indeterminate (for QFT) or failed (for TSPOT.TB) IGRA results. We defined the diagnosis date as the specimen collection date of the first M. tuberculosispositive culture and the time to TB treatment as the number of days from the diagnosis date to the date when the TB treatment started.

\section{Statistical Analysis}

We reported demographic and clinical characteristics as medians and interquartile ranges (IQRs) for continuous variables and as numbers and percentages for categorical variables. We compared the differences between groups (e.g., IGRA-positive group vs. IGRA-negative group) using the Kruskal-Wallis test for continuous variables and the $\chi^{2}$ test for categorical variables. We used logistic regression with the robust standard error option to obtain unbiased standard errors and calculated odds ratios (ORs) and 95\% CIs to identify potential associations between negative IGRA results and demographic, clinical, or laboratory characteristics. We further investigated variables having a $\mathrm{p}$ value of $<0.2$ in the univariate logistic regression by multiple logistic regression modeling to identify characteristics significantly associated with negative IGRA results. We analyzed the survival during the 1-year period after TB diagnosis and stratified by IGRA type using the KaplanMeier method. We compared the survival differences between IGRA types using the log-rank test. We used Cox proportional hazards modeling to determine the risk factors associated with death during the 1-year period after TB diagnosis. We conducted variable selection for the multiple

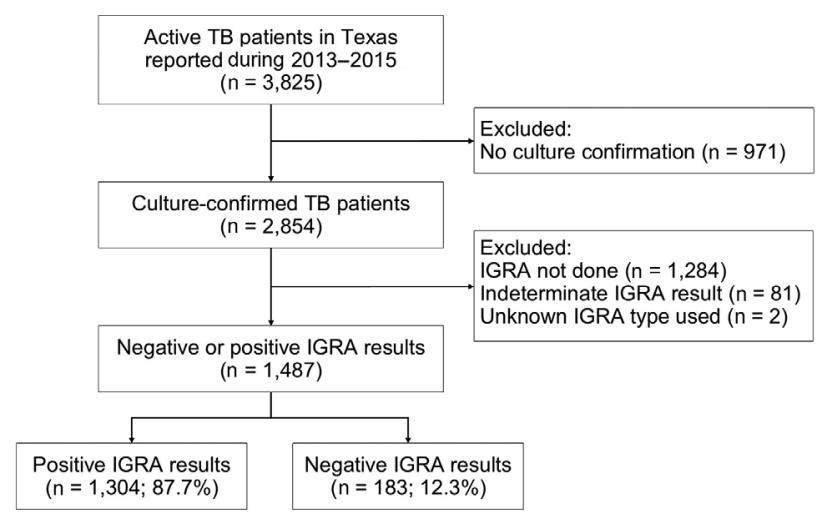

Figure 1. Flowchart showing selection of culture-confirmed TB patients with IGRA results, Texas, USA, 2013-2015. IGRA. IGRA, interferon- $y$ release assay; TB, tuberculosis. logistic regression and Cox proportional hazards models using the Bayesian model averaging method $(14,15)$. In brief, we ran Stata's Bayesian model averaging program to evaluate possible model sets for all variables having a $p$ value of $<0.2$ in the univariate analysis, and Stata suggested good models that included the variables with a high posterior probability of being a risk factor. We used the likelihood ratio test to further reduce the model subsets and selected the best model on the basis of its small Bayesian information criterion. We also tested the selected models for the proportional hazard assumption and evaluated model discrimination using the Harrell $\mathrm{C}$ statistic. We performed all analyses with Stata version 14.2 (StataCorp LLC, College Station, TX, USA) and considered $\mathrm{p}$ values $<0.05$ statistically significant.

\section{Results}

Among the 3,825 patients with TB disease reported in Texas during 2013-2015, a total of 2,854 (74.6\%) were confirmed positive by $M$. tuberculosis culture (Figure 1). Of the 2,854 patients with culture-confirmed TB, 1,527

\begin{tabular}{|c|c|}
\hline \multicolumn{2}{|c|}{$\begin{array}{l}\text { Table 1. Characteristics of 1,487 culture-confirmed TB patients } \\
\text { with negative and positive IGRA results, Texas, USA, 2013- } \\
2015^{*}\end{array}$} \\
\hline Characteristic & Value \\
\hline $\begin{array}{l}\text { Age, } y, \text { median (IQR) } \\
\text { Age } \geq 60 \text { y }\end{array}$ & $\begin{array}{c}47.0(30.0-61.0) \\
402(27.0)\end{array}$ \\
\hline \multicolumn{2}{|l|}{ Sex } \\
\hline M & $942(63.3)$ \\
\hline $\mathrm{F}$ & $545(36.7)$ \\
\hline \multicolumn{2}{|l|}{ Race/Ethnicity } \\
\hline Non-Hispanic white & $152(10.2)$ \\
\hline Black & 275 (18.5) \\
\hline Hispanic & 762 (51.2) \\
\hline Asian & $288(19.4)$ \\
\hline Other & $10(0.7)$ \\
\hline Foreign-born & $897(60.3)$ \\
\hline Resident of long-term care facility & $20(1.3)$ \\
\hline Homeless & $100(6.7)$ \\
\hline Excess alcohol user & $257(17.3)$ \\
\hline Injection drug user & $38(2.6)$ \\
\hline \multicolumn{2}{|l|}{ Chest radiograph result } \\
\hline Normal & $160(10.8)$ \\
\hline Consistent with TB & $1,240(83.4)$ \\
\hline Unknown/Not done & $87(5.9)$ \\
\hline \multicolumn{2}{|l|}{ TB site } \\
\hline Pulmonary & $1,136(76.4)$ \\
\hline Extrapulmonary & $196(13.2)$ \\
\hline Both & $155(10.4)$ \\
\hline \multicolumn{2}{|l|}{ HIV status } \\
\hline Negative & $1,279(86.0)$ \\
\hline Positive & $90(6.1)$ \\
\hline Unknown & $118(7.9)$ \\
\hline MDR & $13(0.9)$ \\
\hline $\begin{array}{l}\text { Days from diagnosis to treatment, median } \\
\text { (IQR) }\end{array}$ & $3.0(0-13.0)$ \\
\hline Died within 1 year of TB diagnosis & $105(7.1)$ \\
\hline $\begin{array}{l}\text { *Values are no. (\%) unless otherwise indicated. } \\
\text { calculated on the basis of the number of patient } \\
\text { IGRA, interferon- } \gamma \text { release assay; IQR, interqua }\end{array}$ & $\begin{array}{l}\text { tages were } \\
\text { ata available. } \\
\text { ge; MDR, multidrug }\end{array}$ \\
\hline
\end{tabular}


(53.5\%) had an IGRA result, with $97.4 \%(1,487 / 1,527)$ having either a positive $(87.7 \%, 1,304 / 1,487)$ or negative $(12.3 \%, 183 / 1,487)$ result. Of the 2,854 culture-confirmed TB patients, 1,367 (47.9\%) were excluded from analysis, either because the IGRA was not done or reported or the IGRA was done but the result was indeterminate or the test type was not specified in the surveillance record (Figure 1). Compared with the patients included in analysis, the patients excluded from analysis were less likely to be Asian and more likely to be men, foreign-born persons, persons who used alcohol in excess or were injection drug users, persons with TB-related radiographic abnormalities, and persons who died (online Technical Appendix
Table, https://wwwnc.cdc.gov/EID/article/24/3/17-1633Techapp1.pdf).

The patients included in the study sample $(\mathrm{N}=1,487)$ had a median age of 47 (IQR 30-61) years, and most were foreign-born $(60.3 \%) ; 63.3 \%$ were men (Table 1). More than half (51.2\%) of the population was Hispanic. Most of the patients had a chest radiograph consistent with TB disease (83.4\%). A total of $105(7.1 \%)$ TB patients died within 1 year of TB diagnosis (Table 1).

Compared with patients having positive IGRA results (median age 46 years), those having negative IGRA results were older (median age 55 years; $p<0.001$ ), typically $\geq 60$ years of age (OR 2.13, 95\% CI 1.55-2.93). Patients

\begin{tabular}{|c|c|c|c|c|}
\hline Variable & $\begin{array}{c}\text { Positive IGRA, } \\
n=1,304\end{array}$ & $\begin{array}{c}\text { Negative IGRA, } \\
n=183\end{array}$ & Crude OR $(95 \% \mathrm{Cl})$ & $\mathrm{p}$ value \\
\hline Age, y, median (IQR) & $46(29-60)$ & $55(40-68)$ & $1.02(1.01-1.03)$ & $<0.001$ \\
\hline Age $\geq 60 y$ & $326(25.0)$ & $76(41.5)$ & $2.13(1.55-2.93)$ & $<0.001$ \\
\hline \multicolumn{5}{|l|}{ Sex } \\
\hline $\mathrm{F}$ & $479(36.7)$ & $66(36.1)$ & Reference & \\
\hline M & $825(63.3)$ & $117(63.9)$ & $1.03(0.75-1.42)$ & 0.861 \\
\hline Non-Hispanic white & $116(8.9)$ & $36(19.7)$ & $2.50(1.66-3.77)$ & $<0.001$ \\
\hline US born & $505(38.7)$ & $85(46.4)$ & $1.37(1.01-1.87)$ & 0.046 \\
\hline Homeless & $85(6.5)$ & $15(8.2)$ & $1.28(0.72-2.27)$ & 0.40 \\
\hline Resident of correction institution & $50(3.8)$ & $4(2.2)$ & $0.56(0.20-1.57)$ & 0.27 \\
\hline Resident of long-term care facility & $18(1.4)$ & $2(1.1)$ & $0.79(0.18-3.43)$ & 0.75 \\
\hline Injection drug user & $35(2.7)$ & $3(1.6)$ & $0.60(0.18-1.99)$ & 0.41 \\
\hline Excessive alcohol user & $218(16.7)$ & $39(21.3)$ & $1.35(0.92-1.98)$ & 0.13 \\
\hline Contact with TB patient within past $2 \mathrm{y}$ & $73(5.6)$ & $3(1.6)$ & $0.28(0.09-0.90)$ & 0.03 \\
\hline Received TNF- $\alpha$ antagonist therapy & $2(0.2)$ & $1(0.5)$ & $3.58(0.32-39.64)$ & 0.30 \\
\hline Solid organ transplant recipient & $1(0.1)$ & $1(0.5)$ & $7.16(0.45-114.96)$ & 0.17 \\
\hline Diabetes & $265(20.3)$ & $39(21.3)$ & $1.06(0.73-1.55)$ & 0.76 \\
\hline Chronic kidney disease & $16(1.2)$ & $3(1.6)$ & $1.34(0.39-4.65)$ & 0.64 \\
\hline Immunosuppression & $30(2.3)$ & $7(3.8)$ & $1.69(0.73-3.90)$ & 0.22 \\
\hline Previous TB & $31(2.4)$ & $1(0.5)$ & $0.23(0.03-1.66)$ & 0.14 \\
\hline \multicolumn{5}{|l|}{ Chest radiograph result } \\
\hline Normal & $137(10.5)$ & $23(12.6)$ & Reference & \\
\hline Consistent with TB & $1,092(83.7)$ & $148(80.9)$ & $0.81(0.50-1.30)$ & 0.38 \\
\hline Unknown/Not done & $75(5.8)$ & $12(6.6)$ & $0.95(0.45-2.02)$ & 0.90 \\
\hline \multicolumn{5}{|l|}{ TB site } \\
\hline Pulmonary & $997(76.5)$ & $139(76.0)$ & Reference & \\
\hline Extrapulmonary & $169(13.0)$ & $27(14.8)$ & $1.15(0.74-1.79)$ & 0.55 \\
\hline Both & $138(10.6)$ & $17(9.3)$ & $0.88(0.52-1.51)$ & 0.65 \\
\hline \multicolumn{5}{|l|}{ HIV status } \\
\hline Negative & $1,134(87.0)$ & $145(79.2)$ & Reference & \\
\hline Positive & $70(5.4)$ & $20(10.9)$ & $2.23(1.32-3.78)$ & 0.003 \\
\hline Unknown/Not done & $100(7.7)$ & $18(9.8)$ & $1.41(0.83-2.39)$ & 0.21 \\
\hline Epidemiologically linked & $118(9.0)$ & $8(4.4)$ & $0.46(0.22-0.96)$ & 0.04 \\
\hline Tested by T-SPOT.TB & $436(33.4)$ & $76(41.5)$ & $1.41(1.03-1.94)$ & 0.03 \\
\hline IGRA sample collected after TB treatment initiated & $415(31.8)$ & $74(40.4)$ & $1.45(1.06-20)$ & 0.02 \\
\hline MDR & $12(0.9)$ & $1(0.5)$ & $0.59(0.08-4.58)$ & 0.62 \\
\hline Genotyped & $1,260(96.6)$ & $175(95.6)$ & $0.76(0.35-1.65)$ & 0.49 \\
\hline East Asian family lineage & $231(17.7)$ & $27(14.8)$ & $0.80(0.52-1.24)$ & 0.32 \\
\hline Clustered & $555(44.1)$ & $85(48.6)$ & $1.20(0.87-1.65)$ & 0.26 \\
\hline GENType G00010 & $34(2.6)$ & $6(3.3)$ & $1.27(0.52-3.06)$ & 0.60 \\
\hline Days from diagnosis to treatment initiation, median (IQR) & $2.0(0-12.0)$ & $5.0(0.5-26.0)$ & $1.00(1.00-1.01)$ & 0.04 \\
\hline Follow-up, mo, median (IQR)† & $9.4(7.3-10.7)$ & $9.2(7.0-10.7)$ & $0.96(0.92-1.01)$ & 0.11 \\
\hline \multicolumn{5}{|l|}{ Year of diagnosis } \\
\hline 2013 & $373(28.6)$ & $56(30.6)$ & Reference & \\
\hline 2014 & $428(32.8)$ & $53(29.0)$ & $0.82(0.55-1.23)$ & 0.35 \\
\hline 2015 & $503(38.6)$ & $74(40.4)$ & $0.98(0.68-1.42)$ & 0.92 \\
\hline
\end{tabular}


Table 3. Multivariate analysis of association between negative IGRA results and culture-confirmed TB patient characteristics, by IGRA type, Texas, USA, 2013-2015*

\begin{tabular}{|c|c|c|c|c|c|c|}
\hline \multirow[b]{2}{*}{ Variable } & \multicolumn{2}{|c|}{ All IGRA results, $\mathrm{N}=1,338 \dagger$} & \multicolumn{2}{|c|}{ QFT only, $\mathrm{n}=875 \dagger$} & \multicolumn{2}{|c|}{ T-SPOT.TB only, $\mathrm{n}=463 \dagger$} \\
\hline & aOR $(95 \% \mathrm{Cl})$ & $p$ value & $\mathrm{aOR}(95 \% \mathrm{Cl})$ & $p$ value & aOR $(95 \% \mathrm{Cl})$ & $p$ value \\
\hline Age in years & $1.02(1.01-1.03)$ & $<0.001$ & $1.02(1.01-1.04)$ & $<0.001$ & $1.02(1.00-1.03)$ & 0.01 \\
\hline Non-Hispanic white race/ethnicity & $2.61(1.70-4.02)$ & $<0.001$ & $2.76(1.60-4.76)$ & $<0.001$ & $2.29(1.11-4.73)$ & 0.03 \\
\hline HIV-positive status & $2.72(1.56-4.77)$ & $<0.001$ & $3.59(1.86-6.94)$ & $<0.001$ & $1.34(0.44-4.07)$ & 0.60 \\
\hline Tested by T-SPOT.TB & $1.58(1.12-2.24)$ & 0.01 & - & - & - & - \\
\hline Time from diagnosis to treatment & $1.00(1.00-1.01)$ & 0.046 & $1.00(1.00-1.01)$ & 0.22 & $1.01(1-1.01)$ & 0.03 \\
\hline
\end{tabular}

with negative results were also more likely than those with positive results to be non-Hispanic white (OR 2.50, 95\% CI 1.66-3.77), US-born (OR 1.37, 95\% CI 1.011.87), and HIV-infected (OR 2.23, 95\% CI 1.32-3.78) (Table 2). Culture-positive, IGRA-negative patients were more likely than culture-positive, IGRA-positive patients to have been tested with the T-SPOT.TB assay (OR 1.41, 95\% CI 1.03-1.94). Culture-positive, IGRA-negative patients had a significantly longer median time from diagnosis to treatment initiation (5 [IQR 0.5-26.0] days) than culture-positive, IGRA-positive patients (2 [IQR $0-12]$ days). The proportion of specific genotype clusters between patients having negative $(48.6 \%)$ and positive (44.1\%) IGRA results was not significantly different ( $\mathrm{p}$ $=0.26$ ). The M. tuberculosis genotype cluster G00010 was the most common found in study patients, with no significant difference found between the negative (3.3\%) and positive $(2.6 \%)$ IGRA groups $(\mathrm{p}=0.60)$ (Table 2$)$. Fewer IGRA-negative patients reported contact with an infectious TB patient during the previous 2 years (OR $0.28,95 \%$ CI $0.09-0.90$ ) and, therefore, were less likely to be epidemiologically linked to a TB patient (OR 0.46, 95\% CI 0.22-0.96). Multivariate analysis results suggested that older age, non-Hispanic white race/ethnicity,
HIV co-infection, being tested with the T-SPOT.TB assay, and longer time to TB treatment were significantly associated with having a negative IGRA result (Table 3). In stratified analyses by IGRA type, a negative QFT result was significantly associated with older age, non-Hispanic white race/ethnicity, and HIV co-infection, and a negative T-SPOT.TB result was significantly associated with older age, non-Hispanic white race/ethnicity, and longer time to TB treatment (Table 3).

Patients with negative IGRA results had a higher overall mortality than those with positive IGRA results both at the time of diagnosis $(2.7 \%$ [5/183] vs. $0.7 \%$ [9/1,304]; crude OR 4.04, 95\% CI 1.34-12.2) and during TB treatment $(11.2 \%$ [20/178] vs. $4.9 \%$ [64/1,295]; crude OR $2.43,95 \%$ CI 1.43-4.13). The 1-year survival rate from diagnosis was $83 \%$ for patients with negative IGRA results and $94 \%$ for patients with positive IGRA results $(\mathrm{p}<0.001)$ (Figure 2), with an adjusted hazard ratio of 1.99 (95\% CI 1.18-3.33; $\mathrm{p}=0.01$ ) (Table 4$)$. When stratified by IGRA type, only negative T-SPOT.TB results remained significantly associated with death. Older age, HIV co-infection, and time to TB treatment were identified by Cox proportional hazards modeling as independent risk factors for death; however, only age remained significant after stratifying by IGRA

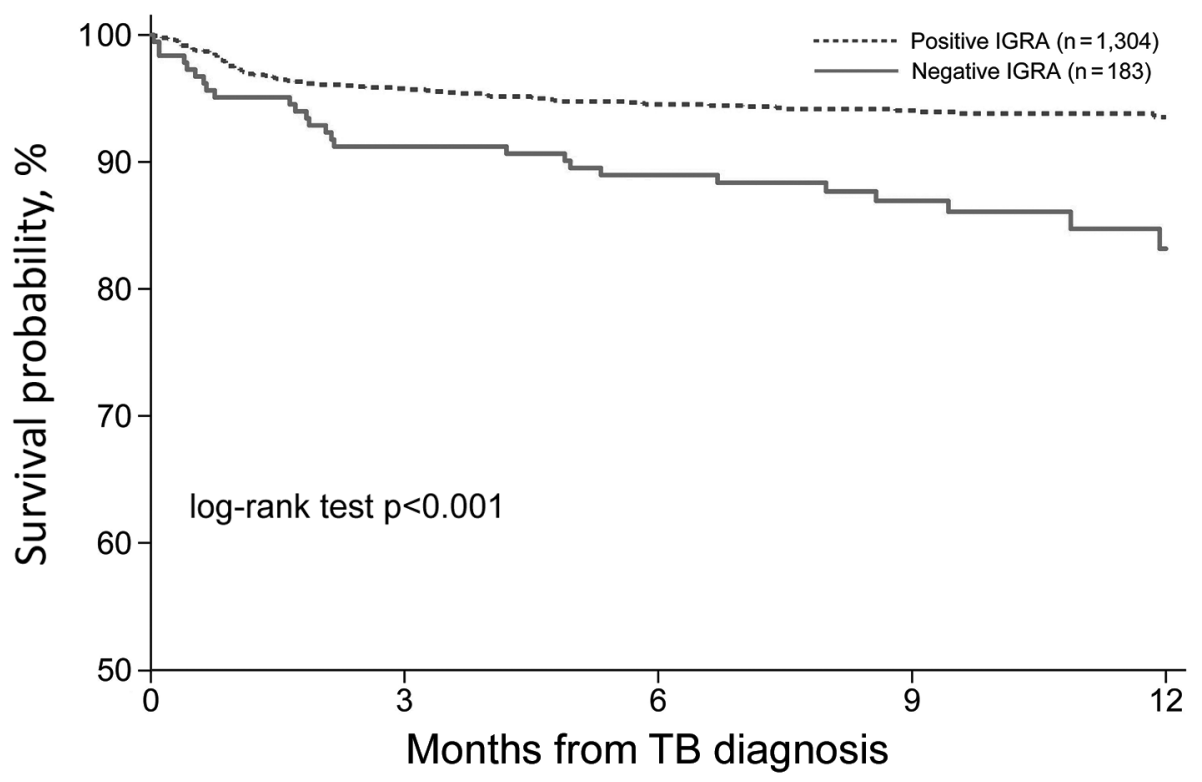

Figure 2. One-year survival from date of TB diagnosis for cultureconfirmed TB, stratified by IGRA result, Texas, USA, 2013-2015. IGRA. IGRA, interferon- $y$ release assay; TB, tuberculosis. 
Table 4. Cox proportional hazards model for association between 1-year survival from date of tuberculosis diagnosis and characteristics of culture-confirmed TB patients with negative IGRA results, by IGRA type, Texas, USA, 2013-2015*

\begin{tabular}{|c|c|c|c|c|c|c|}
\hline \multirow[b]{2}{*}{ Variable } & \multicolumn{2}{|c|}{ All IGRA results, $\mathrm{N}=1,343 \dagger$} & \multicolumn{2}{|c|}{ QFT only, $\mathrm{n}=880 \dagger$} & \multicolumn{2}{|c|}{ T-SPOT.TB only, $\mathrm{n}=463 \dagger$} \\
\hline & aHR $(95 \% \mathrm{Cl})$ & $p$ value & aHR $(95 \% \mathrm{Cl})$ & $p$ value & aHR $(95 \% \mathrm{Cl})$ & $p$ value \\
\hline Negative IGRA result & $1.99(1.18-3.33)$ & 0.01 & $1.64(0.88-3.08)$ & 0.120 & $3.36(1.27-8.90)$ & 0.02 \\
\hline Age in years & $1.06(1.04-1.07)$ & $<0.001$ & $1.06(1.04-1.08)$ & $<0.001$ & $1.05(1.02-1.08)$ & 0.001 \\
\hline Alcohol & $1.55(0.92-2.60)$ & 0.10 & $1.75(0.97-3.15)$ & 0.06 & $1.19(0.39-3.63)$ & 0.76 \\
\hline HIV positive & $5.00(2.68-9.33)$ & $<0.001$ & $4.79(2.43-9.47)$ & $<0.001$ & $4.38(0.90-21.44)$ & 0.07 \\
\hline Time from diagnosis to treatment & $0.99(0.99-1.00)$ & 0.002 & $0.99(0.99-1.00)$ & 0.01 & $0.99(0.98-1.00)$ & 0.15 \\
\hline
\end{tabular}

type (Table 4). The 1-year mortality did not differ significantly by year of TB diagnosis $(9.1 \%$ for $2013,6.0 \%$ for 2014 , and $6.4 \%$ for $2015 ; p=0.15$ ) (data not shown). Multivariate Cox modeling assessing the survival after the initiation of TB treatment also suggested that negative IGRA results were significantly associated with a higher 1-year mortality (Table 5).

\section{Discussion}

Our results suggest that TB patients with false-negative IGRA results had significantly poorer outcomes, with nearly 2 times the odds for death within 1 year of TB diagnosis, compared with patients with positive IGRA results. Falsenegative IGRA results were more likely to occur in older, non-Hispanic white, and HIV-infected patients or patients tested with the T-SPOT.TB assay.

Our study not only confirms the World Health Organization and Centers for Disease Control and Prevention recommendation of not using IGRAs as rule-out tests for TB disease $(7,16)$ but also indicates that patients with confirmed TB diagnoses but negative IGRA results have poor outcomes. Our study results highlight the need for having a systematic and extensive management strategy for suspected TB patients who have negative IGRA results to minimize misdiagnosis and improve patient outcomes. In symptomatic patients being evaluated for TB disease, further diagnostic evaluation and close follow-up for TB disease should be considered, especially in persons $\geq 60$ years of age, non-Hispanic white, HIV-infected, or tested by the T-SPOT.TB assay.

Although false-negative IGRA results were associated with a nearly 2 -fold increase in risk for death within 1 year of TB diagnosis, only a false-negative T-SPOT.TB result remained significantly associated with death after stratification by IGRA type. This finding suggests that patients who had false-negative T-SPOT.TB results in our study might be sicker than those who had false-negative QFT results. This explanation is consistent with the significant delay in treatment initiation after TB diagnosis, especially for patients in the T-SPOT.TB group. The significant association between time to treatment and death among all patients and patients by IGRA type suggests that delayed treatment might contribute to the higher mortality of patients with false-negative IGRA results. This finding demonstrates the programmatic implications for treating patients who have negative IGRA findings. Consistent with previously published data, we also found a significantly higher risk for death among patients of older age or with HIV co-infection (17-19).

Consistent with results of previous studies $(8-10,20)$, in our study, older age was also significantly associated with a false-negative result for both the QFT and T-SPOT. $T B$ assays. An explanation for this association could be the gradual decrease of IFN- $\gamma$ production that occurs in response to M. tuberculosis-specific antigens ESAT-6 and CFP-10 with age $(9,10)$. Other authors have observed a higher rate of false-negative IGRA results in young children (21-23). However, all 17 children $\leq 5$ years of age in our study had positive IGRA results.

HIV co-infection was associated with a false-negative QFT result but not a false-negative T-SPOT.TB result. This finding is consistent with the current literature, which has suggested that the T-SPOT.TB assay has higher sensitivity than QFT among HIV co-infected persons, especially

Table 5. Cox proportional hazards model for association between 1-year survival from tuberculosis treatment start date and characteristics of culture-confirmed TB patients with negative IGRA results, by IGRA type, Texas, USA, 2013-2015*

\begin{tabular}{|c|c|c|c|c|c|c|}
\hline \multirow[b]{2}{*}{ Variable } & \multicolumn{2}{|c|}{ All IGRA results, $\mathrm{N}=1,343 \dagger$} & \multicolumn{2}{|c|}{ QFT only, $n=880 \dagger$} & \multicolumn{2}{|c|}{ T-SPOT. TB only, $n=463+$} \\
\hline & $\operatorname{aHR}(95 \% \mathrm{Cl})$ & $\mathrm{p}$ value & $\operatorname{aHR}(95 \% \mathrm{Cl})$ & $\mathrm{p}$ value & $\operatorname{aHR}(95 \% \mathrm{Cl})$ & $\mathrm{p}$ value \\
\hline Negative IGRA result & $2.12(1.24-3.63)$ & 0.01 & $1.84(0.98-3.47)$ & 0.06 & $3.41(1.18-9.88)$ & 0.02 \\
\hline Age in years & $1.06(1.04-1.07)$ & $<0.001$ & $1.06(1.04-1.07)$ & $<0.001$ & $.02-1.09)$ & 0.002 \\
\hline Alcohol use & $1.42(0.82-2.48)$ & 0.21 & $1.57(0.85-2.93)$ & 0.15 & $.30-3.80)$ & 0.92 \\
\hline HIV positive & $4.68(2.40-9.12)$ & $<0.001$ & $4.60(2.26-9.34)$ & $<0.001$ & $2.75(0.32-23.40)$ & 0.36 \\
\hline Time from diagnosis to treatment & $0.99(0.99-1.00)$ & 0.002 & 0.99 (0.99-1.00) & 0.01 & $0.99(0.98-1.00)^{\prime}$ & 0.10 \\
\hline
\end{tabular}

*aHR, adjusted hazard ratio; IGRA, interferon-y release assay; QFT, QuantiFERON-TB Gold In-Tube; TB, tuberculosis.

†Cox proportional hazards models were run with data from patients having complete data sets for all the included variables; aHRs were from the multivariate Cox proportional hazards models. 
those with low CD4+ T-cell counts $(24,25)$. As observed in a previous study (26), in our study, non-Hispanic white race/ethnicity was also associated with a false-negative QFT result. In our analysis, extrapulmonary TB was not significantly associated with a false-negative IGRA result or patient outcomes.

Our study has several limitations. We could not completely rule out some provider selection bias, considering nearly $48 \%(1,367 / 2,854)$ of the culture-confirmed TB patients were excluded from the analysis, most (94\%, $1,284 / 1,367)$ because an IGRA was not performed. Because we used deidentified administrative data obtained from NEDSS, quantitative test results for QFT and TSPOT.TB were not available for analytic evaluation. In addition, by selecting only nonindeterminate results (for QFT) and nonfailed results (for T-SPOT.TB), the IGRAs' true sensitivities were overestimated $(89.0 \%$ [868/975] for QFT and 85.2\% [436/512] for T-SPOT.TB; $\mathrm{p}=0.031$ ). If indeterminate and failed results are included with the negative results when measuring the sensitivity, then the true sensitivity of the QFT and TSPOT.TB assays in this population-based screening becomes $84.4 \%$ for QFT and $80.9 \%$ for T-SPOT.TB, and the difference in the sensitivity between QFT and T-SPOT.TB becomes nonsignificant ( $\mathrm{p}$ $=0.095$ ). Immunologic and genetic details, which can help explain the cause of false-negative IGRA results, were also unavailable. Last, because some of the data were originally obtained from interviewing TB patients, the possibility of recall bias cannot be ruled out.

In conclusion, we identified negative IGRA results as a significant predictor for death within 1 year of TB diagnosis among culture-confirmed $M$. tuberculosis-infected patients. Older age, non-Hispanic white race/ethnicity, HIV co-infection, T-SPOT.TB assay results, and longer time to TB treatment were significantly associated with false-negative IGRA results. Healthcare providers should consider these risk factors when making decisions on whether to initiate further diagnostic evaluations for TB patients with negative IGRA results.

\section{Acknowledgments}

The authors acknowledge the selfless work of public health officials and staff at the City of Houston Bureau of Tuberculosis Control, Houston Department of Health \& Human Services; Harris County Public Health; and the TB Elimination Program, Texas Department of State Health Services, who made the data available for use in this analysis.

\section{About the Author}

Dr. Nguyen is an epidemiologist at the Houston Methodist Research Institute in Houston, Texas, USA. His research interests include tuberculosis epidemiology and diagnosis.

\section{References}

1. Centers for Disease Control and Prevention. Interferon-gamma release assays (IGRAs) — blood tests for TB infection. 2017 Aug 17 [cited 2017 Sept 2]. https://www.cdc.gov/tb/publications/factsheets/ testing/igra.htm

2. Texas Department of State Health Services. Texas tuberculosis work plan. Austin (TX): The Department; 2017 Aug 31 [cited 2017 Sept 02]. http://www.dshs.texas.gov/IDCU/disease/tb/policies/ TBWorkPlan.pdf

3. Sester M, Sotgiu G, Lange C, Giehl C, Girardi E, Migliori GB, et al. Interferon- $\gamma$ release assays for the diagnosis of active tuberculosis: a systematic review and meta-analysis. Eur Respir J. 2011;37:100-11. http://dx.doi.org/10.1183/09031936.00114810

4. Laurenti P, Raponi M, de Waure C, Marino M, Ricciardi W, Damiani G. Performance of interferon- $\gamma$ release assays in the diagnosis of confirmed active tuberculosis in immunocompetent children: a new systematic review and meta-analysis. BMC Infect Dis. 2016;16:131. http://dx.doi.org/10.1186/s12879-016-1461-y

5. Lavender TW, Barrett A, Magee J, Ong EL. Interferon- $\gamma$ release assays in the diagnosis of active tuberculosis disease in a lowincident setting: a 5-year review of data. Clin Microbiol Infect. 2013;19:1078-81. http://dx.doi.org/10.1111/1469-0691.12129

6. Metcalfe JZ, Everett CK, Steingart KR, Cattamanchi A, Huang L, Hopewell $\mathrm{PC}$, et al. Interferon- $\gamma$ release assays for active pulmonary tuberculosis diagnosis in adults in low- and middle-income countries: systematic review and meta-analysis. J Infect Dis. 2011;204(Suppl 4):S1120-9. http://dx.doi.org/10.1093/ infdis/jir410

7. World Health Organization. Use of tuberculosis interferon-gamma release assays (IGRAs) in low- and middle-income countries. Geneva: The Organization; 2011.

8. de Visser V, Sotgiu G, Lange C, Aabye MG, Bakker M, Bartalesi F, et al. False-negative interferon- $\gamma$ release assay results in active tuberculosis: a TBNET study. Eur Respir J. 2015;45:279-83. http://dx.doi.org/10.1183/09031936.00120214

9. Hang NT, Lien LT, Kobayashi N, Shimbo T, Sakurada S, Thuong PH, et al. Analysis of factors lowering sensitivity of interferon $-\gamma$ release assay for tuberculosis. PLoS One. 2011;6:e23806. http://dx.doi.org/10.1371/journal.pone.0023806

10. Kobashi Y, Mouri K, Yagi S, Obase Y, Miyashita N, Okimoto N, et al. Clinical utility of the QuantiFERON TB-2G test for elderly patients with active tuberculosis. Chest. 2008;133:1196-202. http://dx.doi.org/10.1378/chest.07-1995

11. Kobashi Y, Shimizu H, Ohue Y, Mouri K, Obase Y, Miyashita N, et al. False negative results of QuantiFERON TB-2G test in patients with active tuberculosis. Jpn J Infect Dis. 2009;62:300-2.

12. Division for Prevention and Preparedness, Texas Department of State Health Services. National Electronic Disease Surveillance System (NEDSS). 2017 Jun 20 [cited 2017 Sep 15]. http://www.dshs.texas.gov/nedss/

13. Ghosh S, Moonan PK, Cowan L, Grant J, Kammerer S, Navin TR. Tuberculosis genotyping information management system: enhancing tuberculosis surveillance in the United States. Infect Genet Evol. 2012;12:782-8. http:/dx.doi.org/10.1016/ j.meegid.2011.10.013

14. Maldonado G, Greenland S. Simulation study of confounderselection strategies. Am J Epidemiol. 1993;138:923-36. http://dx.doi.org/10.1093/oxfordjournals.aje.a116813

15. Wasserman L. Bayesian model selection model averaging. J Math Psychol. 2000;44:92-107. http:/dx.doi.org/10.1006/ jmps.1999.1278

16. Mazurek GH, Jereb J, Vernon A, LoBue P, Goldberg S, Castro K; IGRA Expert Committee; Centers for Disease Control and Prevention. Updated guidelines for using interferon gamma release assays to detect Mycobacterium tuberculosis infection-United States, 2010. MMWR Recomm Rep. 2010;59(RR-5):1-25. 
17. Cruz-Hervert LP, García-García L, Ferreyra-Reyes L, Bobadilla-del-Valle M, Cano-Arellano B, Canizales-Quintero S, et al. Tuberculosis in ageing: high rates, complex diagnosis and poor clinical outcomes. Age Ageing. 2012;41:488-95. http://dx.doi.org/10.1093/ageing/afs028

18. Salvadó M, Garcia-Vidal C, Vázquez P, Riera M, Rodríguez-Carballeira M, Martínez-Lacasa J, et al. Mortality of tuberculosis in very old people. J Am Geriatr Soc. 2010;58:18-22. http://dx.doi.org/10.1111/j.1532-5415.2009.02619.x

19. Negin J, Abimbola S, Marais BJ. Tuberculosis among older adults - time to take notice. Int J Infect Dis. 2015;32:135-7. http://dx.doi.org/10.1016/j.ijid.2014.11.018

20. Liao CH, Lai CC, Tan CK, Chou CH, Hsu HL, Tasi TH, et al. False-negative results by enzyme-linked immunospot assay for interferon- $\gamma$ among patients with culture-confirmed tuberculosis. J Infect. 2009;59:421-3. http://dx.doi.org/10.1016/ j.jinf.2009.09.012

21. Bergamini BM, Losi M, Vaienti F, D'Amico R, Meccugni B, Meacci M, et al. Performance of commercial blood tests for the diagnosis of latent tuberculosis infection in children and adolescents. Pediatrics. 2009;123:e419-24. http://dx.doi.org/ 10.1542/peds.2008-1722

22. Grare M, Derelle J, Dailloux M, Laurain C. QuantiFERON-TB Gold In-Tube as help for the diagnosis of tuberculosis in a French pediatric hospital. Diagn Microbiol Infect Dis. 2010;66:366-72. http://dx.doi.org/10.1016/j.diagmicrobio.2009.11.002
23. Kampmann B, Whittaker E, Williams A, Walters S, Gordon A, Martinez-Alier $N$, et al. Interferon- $\gamma$ release assays do not identify more children with active tuberculosis than the tuberculin skin test. Eur Respir J. 2009;33:1374-82. http://dx.doi.org/ 10.1183/09031936.00153408

24. Aabye MG, Ravn P, PrayGod G, Jeremiah K, Mugomela A, Jepsen M, et al. The impact of HIV infection and CD4 cell count on the performance of an interferon gamma release assay in patients with pulmonary tuberculosis. PLoS One. 2009;4:e4220. http://dx.doi.org/10.1371/journal.pone.0004220

25. Chen J, Zhang R, Wang J, Liu L, Zheng Y, Shen Y, et al. Interferon-gamma release assays for the diagnosis of active tuberculosis in HIV-infected patients: a systematic review and meta-analysis. PLoS One. 2011;6:e26827. http://dx.doi.org/ 10.1371/journal.pone.0026827

26. Mazurek GH, Weis SE, Moonan PK, Daley CL, Bernardo J, Lardizabal AA, et al. Prospective comparison of the tuberculin skin test and 2 whole-blood interferon- $\gamma$ release assays in persons with suspected tuberculosis. Clin Infect Dis. 2007;45:837-45. http://dx.doi.org/10.1086/521107

Address for correspondence: Edward A. Graviss, Houston Methodist Research Institute, Department of Pathology and Genomic Medicine, Mail Station R6-414, 6670 Bertner, Houston, TX 77030, USA; email: eagraviss@houstonmethodist.org

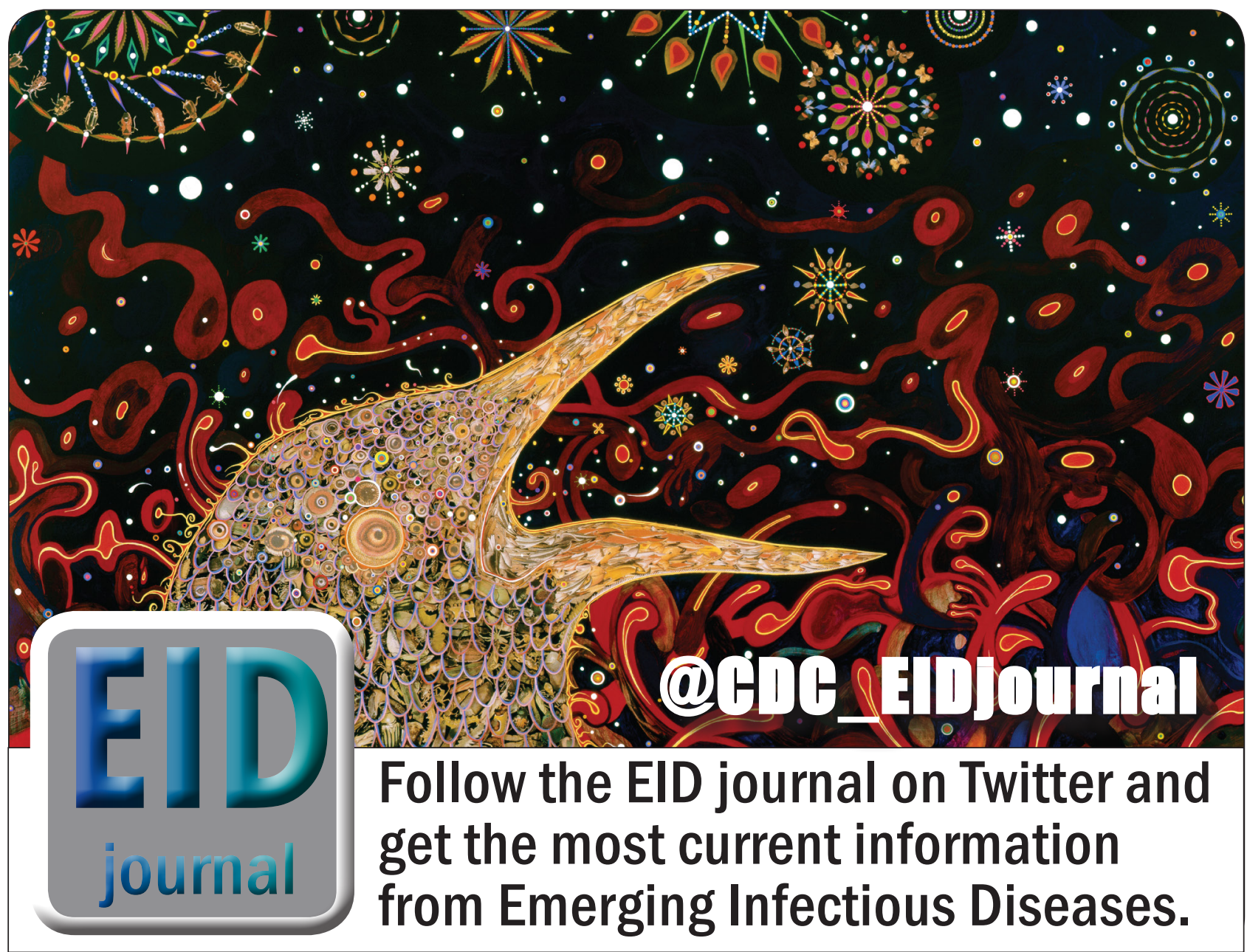

